\title{
A data set linking large-scale, individual semantic networks and cognitive performance
}

\author{
Dirk U. Wulff ${ }^{1,2}$, Samuel Aeschbach ${ }^{1}$, Simon De Deyne ${ }^{3}$, and Rui Mata ${ }^{1,2}$ \\ ${ }^{1}$ University of Basel \\ ${ }^{2}$ Max Planck Institute for Human Development \\ ${ }^{3}$ University of Melbourne
}

\begin{abstract}
We report data from a proof-of-concept study involving the concurrent assessment of largescale individual semantic networks and cognitive performance. The data include 10,800 free associations-collected using a dedicated web-based platform over the course of 2-4 weeks-and responses to several cognitive tasks, including verbal fluency, episodic memory, associative recall tasks, from four younger and four older native German speakers. The data are unique in scope and composition and shed light on individual and age-related differences in mental representations and their role in cognitive performance across the lifespan.
\end{abstract}

Keywords: semantic networks, cognitive aging, individual differences

\section{Collection Date}

The data were collected from August to October 2018.

\section{Background}

Over the lifespan, people accumulate a large and idiosyncratic set of experiences that shape their mental knowledge representations. These changes in mental representations driven by experience could potentially be a major factor underlying typical age-related patterns, such as decreased memory performance with increased age (Buchler \& Reder, 2007, Ramscar et al., 2014, Wulff et al., 2019). In line with this view, recent research (Kenett et al., 2020; Siew et al., 2019) has documented consistent differences in the size and structure of younger and older adults' mental representations (Dubossarsky et al., 2017; Wulff et al., 2018, October 29). To evaluate whether and how strongly these differences in representations contribute to differences in $\operatorname{cog}$ nitive performance across age, we designed the My Small Word of Words (MySWOW) project. Building on ongoing efforts to obtain word association norms for several languages in a large online citizen-science project, the Small

Dirk U. Wulff (10 https://orcid.org/0000-0002-4008-8022 Samuel Aeschbach (D) https://orcid.org/0000-0002-6167-4901 Simon De Deyne (D) https://orcid.org/0000-0002-7899-6210 Rui Mata (D) https://orcid.org/0000-0002-1679-906X

Correspondence concerning this article should be addressed to Dirk Wulff, Department of Psychology, University of Basel, Missionsstrasse 60-62, 4055 Basel, Switzerland. E-mail: dirk.wulff@gmail.com
World of Words (SWOW; e.g., De Deyne et al., 2019) study (https://smallworldofwords.org), MySWOW aims to elicit large-scale, free association networks from single individuals and concurrently assess their cognitive performance across a variety of tasks that are known to be linked to semantic representations. MySWOW addresses shortcomings of previous research, which either had focused on group-level representations (Dubossarsky et al., 2017) or did not concurrently assess cognitive performance on a broad scale (Wulff et al., 2018, October 29). We present data of a proof-of-concept study of MySWOW involving four younger and four older individuals. For additional details of the study rationale, see Wulff et al. (2021, February 15).

\section{Methods}

The MySWOW proof-of-concept study relied on a correlational design encompassing the concurrent assessment of a large number of free word associations and a broad battery of cognitive tasks for four younger and four older individuals. The free association task and cognitive battery were designed to match each other in order to facilitate a comparison of semantic networks and cognitive performance.

\section{Participants}

Four older adults aged 68 to 70 years old and four younger adults aged 24 to 28 years old participated and completed the study. Three more participants began the study, but dropped out after .5\%, $18.7 \%$, and $41.7 \%$ of the free association task. We only report data for the eight participants with complete data. Participants were recruited from the participant pool of the Center for Cognitive and Decision Sciences (CDS) of 
the University of Basel. They were contacted via phone and completed an initial screening to confirm the following inclusion criteria: mother tongue being German or Swiss German, daily access to a computer with a stable Internet connection, absence of neurological or psychiatric diagnoses. Participants were compensated with a flat fee of CHF 220 (USD 245) consisting of CHF 180 for the free association task (CHF 0.05 per cue) and CHF 60 for four hours of laboratory assessment and instructions (CHF 15/h). Participants were compensated with $\mathrm{CHF} 220$ for their full participation consisting of CHF 180 for 3,600 answered cues (CHF 0.05 per cue) and CHF 40 for two to three hours of laboratory assessment and instructions (approx. CHF15/h).

All data were recorded in reference to a random six letter identifier assigned to participants at the beginning of the study. Identifying information such as names or addresses was not recorded. Potentially identifying information such as participants' age, birthday, and profession were not included in the publicly available files. Participants provided informed consent that included permission for public sharing of the data. The study was approved by the internal review board of the Department of Psychology at the University of Basel (\# 014-17-1).

\section{Materials}

\section{Free association task}

Free associations were collected via a password-protected web-based platform that participants could access from home. In the association task, participants were sequentially presented with a total of 3,600 cues for which they provided three associations each, following the same procedure used in SWOW. Participants were instructed to enter, using the keyboard, the first three words that came to mind when thinking about the cue. If fewer than three words came to mind or if the cue was not recognized, the participant could proceed to the next cue by clicking on a "no further responses" or "unknown word" button, respectively. Figure 1 shows a screenshot of the free association interface.

The 3,600 cues consisted of 3,000 unique and 600 repeated cues. The 3,000 unique cues, in turn, consisted of three subsets of 1,000 cues each. To ensure high coverage of central words in people's semantic networks, the first subset consisted of 1,000 highest frequency words among the 4,500 cue words that, at time, were included in the German SWOW, with frequency determined using the German SUBTLEX frequency norms (Brysbaert et al., 2011). To ensure high coverage of the connections within people's networks, the second subset consisted of those 1,000 from the remaining 3,500 cues in the German SWOW that most likely produced one of the cues in the first subset. Finally, to ensure a high network depth, the third subset consisted of the 1,000 most frequent associates in the German SWOW given to the

\section{Beantwortet: 0/15 Verdient: $0.00 \mathrm{CHF}$ E Büroklammer}

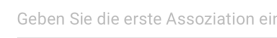

+ Nächste Eingabe $\quad \times$ Unbekanntes Wort

Figure 1

Screenshot of the free association task. The screenshot shows one trial in training mini-study requiring associations to the cue "Büroklammer" (paper clip).

cues of the first subset. The cues were presented to the participants in the same fixed, randomly determined order.

Responses were cleaned in the following way. First, all responses matching either individual words or composites of words included in the German aspell dictionary were accepted as valid. The remaining words were subjected to manual correction. Overall, $4.2 \%$ of responses were corrected manually with a median string edit distance (i.e., the number of letters that were changed) of 2 (mean $=2.42$ ).

\section{Cognitive assessment}

The cognitive battery consisted of two sets of tasks fulfilling different purposes. The purpose of the first set was the assessment of people's general cognitive abilities and functioning. This set included a 20-minute timed version of the Advanced Progressive Matrices (APM; Hamel \& Schmittmann, 2006) as a measure of general intelligence, a digit-symbol substitution test, as is found in the Wechsler Adult Intelligence Scale IV as subtest "coding" (WAIS-IV; Wechsler, 2008) as a measure of processing speed, the MehrfachwahlWortschatz-Intelligenztest: Form I (MWT-A; Lehrl et al., 1995) as a measure of vocabulary size, and, finally, the DemTect (Kalbe et al., 2004) as a screen for dementia. The purpose of the second set was to establish word-level links between the free association network and cognitive performance. This set included 10-minute category (animals) and phonemic fluency (letter S) fluency tasks (e.g., Wulff et al., 2018, October 29), an episodic list memory task modeled after Penn Electrophysiology of Encoding and Retrieval Study (e.g., Healey \& Kahana, 2016), and an associative recall task modeled after Naveh-Benjamin et al. (2003). Behavior in the two fluency tasks can be related to the free association network based on the fact that both cues and responses naturally 
Table 1

Tasks in the cognitive battery

\begin{tabular}{|c|c|c|c|}
\hline Task & Description & Motivation & Reference \\
\hline Category fluency & $\begin{array}{l}\text { Name all the animals you can in } 10 \\
\text { minutes. }\end{array}$ & $\begin{array}{l}\text { Predict performance } \\
\text { from network }\end{array}$ & $\begin{array}{l}\text { Wulff et al. } 2018, \text { Oc- } \\
\text { tober } 29)\end{array}$ \\
\hline Phonemic fluency & $\begin{array}{l}\text { Name all words starting with letter } S \\
\text { you can in } 10 \text { minutes. }\end{array}$ & $\begin{array}{l}\text { Predict performance } \\
\text { from network }\end{array}$ & Griffiths et al. (2007) \\
\hline Episodic memory task & $\begin{array}{l}\text { Study a word list and then recall the } \\
\text { words in any order ( } 20 \text { lists, } 16 \text { words } \\
\text { per list). }\end{array}$ & $\begin{array}{l}\text { Predict performance } \\
\text { from network }\end{array}$ & $\begin{array}{l}\text { Healey and Kahana } \\
(2016)\end{array}$ \\
\hline Associative recall task & $\begin{array}{l}\text { Study a list of word pairs, then recall } \\
\text { for each one word of a pair while be- } \\
\text { ing cued with the other ( } 4 \text { lists, of } 16 \\
\text { word pairs). }\end{array}$ & $\begin{array}{l}\text { Predict performance } \\
\text { from network }\end{array}$ & $\begin{array}{l}\text { Naveh-Benjamin et al. } \\
(2003)\end{array}$ \\
\hline $\begin{array}{l}\text { Advanced Progressive Ma- } \\
\text { trices }\end{array}$ & Solve abstract reasoning problems. & $\begin{array}{l}\text { General cognitive abili- } \\
\text { ties }\end{array}$ & $\begin{array}{l}\text { Hamel and } \\
\text { Schmittmann (2006) }\end{array}$ \\
\hline Digit-symbol substitution & $\begin{array}{l}\text { Assign digits to symbols according to } \\
\text { rule. }\end{array}$ & $\begin{array}{l}\text { General cognitive abili- } \\
\text { ties }\end{array}$ & Wechsler $(2008)$ \\
\hline $\begin{array}{l}\text { Mehrfachwahl-Wortschatz- } \\
\text { Intelligenztest }\end{array}$ & $\begin{array}{l}\text { Recognize words in list of words and } \\
\text { non-words. }\end{array}$ & $\begin{array}{l}\text { General cognitive abili- } \\
\text { ties }\end{array}$ & Lehrl et al. $(1995)$ \\
\hline DemTect & Various cognitive tasks. & $\begin{array}{l}\text { Screen for age-related } \\
\text { pathologies }\end{array}$ & Kalbe et al. (2004) \\
\hline
\end{tabular}

included animals and words starting with the letters S. Participants retrieved between 62 and 113 animals and between 45 and 138 words of the letter S. The retrieved animals overlapped with $1.5 \%$ of cues and $0.8 \%$ of responses, whereas the retrieved words starting with the letter S overlapped with $11.1 \%$ of cues and $11.9 \%$ of responses. The episodic memory task and the associative recall task were populated with nouns from the cue set to establish comparability with the associative network. In the episodic memory task, a total of 20 lists of 16 words each were studied and subsequently recalled. Participants correctly recalled between $28.7 \%$ and $60.9 \%$ of words, with an additional $1.3 \%$ to $25 \%$ intrusions. In the associative recall task, 4 lists consisting of 16 wordpairs were presented and tested. Participants correctly recalled between $32.8 \%$ and $96.8 \%$ of pairs. See also Table 1 for an overview of tasks included in the cognitive assessment in the MySWOW proof-of-concept study.

\section{Entry and debriefing questionnaires}

At study entry, participants provided demographic information concerning their primary language (German or Swiss German), their current profession, their highest academic degree, and the income level of their household. Participants further answered questions on their usual reading behavior, e.g., the number of books read in a year. At debriefing, participants were asked to provide information on their observations during the study, for example, whether they were able to sustain concentration while working on the free associations. The specific questions are reported in the code book (see Table 2).

\section{Procedure}

Participants passing the initial screening over the phone were invited to to our laboratory at the University of Basel for an introductory session lasting approximately 30 minutes. During this session participants provided informed consent, completed the entry questionnaire, and were introduced to the web-based platform using a training mini-study involving 15 cues. Over the course of the next weeks, participants were instructed to $\log$ in and work on the free association task twice a day for 30 minutes each. On average, participants completed the free association task in 26.1 hours spread over 39.4 days. After completing the free association task, participants were invited back to the laboratory for a three-hour session that included the cognitive assessments and study debriefing.

The cognitive assessment and study debriefing session consisted of the following elements: First, participants filled out the debriefing questionnaire. Next, the verbal fluency tasks were conducted orally and recorded for later transcription by two student assistants responsible for data collection. Following the verbal fluency tasks, the participants were administered a 90-second timed Digit Symbol Substitution Test in paper and pencil format. To conclude the first part of the lab session, the Associative Recall task was completed as a computerized task implemented in E-Prime (Psychology Software Tools, Inc., 2016) at a lab-computer. After a 10- 
Table 2

\begin{tabular}{ll} 
Description of Data Files & \\
\hline File & Description \\
\hline participants.csv & $\begin{array}{l}\text { Contains data on demographic } \\
\text { information, reading behavior, } \\
\text { debriefings survey, and all but } \\
\text { four cognitive assessments. } \\
\text { Contains the corrected and } \\
\text { uncorrected free association } \\
\text { data. } \\
\text { associations.csv }\end{array}$ \\
Contains the episodic memory \\
training and test data. \\
Contains the associative recall \\
training and test data. \\
associative_recall.csv \\
Contains animal fluency re- \\
sponse sequences. \\
Contains letter fluency re- \\
letter_fluency.csv
\end{tabular}

minute break, the second part of the lab session began with the List Memory task, which was also implemented as a computerized task using E-Prime (Psychology Software Tools, Inc., 2016). The Mehrfachwahl-Wortschatz-Intelligenztest (MWT-A) was then conducted in paper and pencil format followed by a 20-minute timed version of the Advanced Progressive Matrices (APM) in paper and pencil format. The lab session concluded with the interactive verbal administration of the DemTect, carried out by one of the student assistants. Subsequently, participants received their monetary compensation for participation.

\section{Dataset description}

Table 2 provides an overview of the different files containing the data. All data are available as comma-separated files. A codebook.pdf file provides descriptions of all variable names across the data files. All variable names and data labels have been translated to English. The association and fluency data, however, were not translated.

The data were published on the Open Science Framework (10.17605/OSF.IO/VKWPS) on February 15.02.2021. The data are licensed under Creative Commons AttributionShareAlike 4.0 International (CC BY-SA 4.0).

\section{Reuse potential}

The reported data present the only publicly available resource containing large-scale free-association data on the individual level (cf. Morais et al., 2013). These data are amenable to network analytic (Siew et al., 2019) and traditional approaches to free association data (Nelson et al., 2001 ) that can shed light on individual and age-related differences in semantic representations and retrieval. Of particular value is the fact that the large-scale free association data are accompanied by a diverse cognitive battery, including four tasks that can be linked to the free association data. Further assessment of these links, for instance, using better inference of the underlying network representation or more elaborate models of cognitive performance, promises to improve the understanding of experience-driven differences in mental representations that may contribute to differences in cognitive performance.

\section{Acknowledgements}

We thank Alina Gerlach for helping collecting the data. We thank Laura Wiles for editing the manuscript. This work was supported by a grant from the Swiss Science Foundation (100015_197315) to Dirk U. Wulff.

\section{References}

Brysbaert, M., Buchmeier, M., Conrad, M., Jacobs, A. M., Bölte, J., \& Böhl, A. (2011). The word frequency effect. Experimental Psychology, 58, 412-424.

Buchler, N. E. G., \& Reder, L. M. (2007). Modeling agerelated memory deficits: A two-parameter solution. Psychology and aging, 22(1), 104-121.

De Deyne, S., Navarro, D. J., Perfors, A., Brysbaert, M., \& Storms, G. (2019). The "small world of words" english word association norms for over 12,000 cue words. Behavior research methods, 51(3), 9871006.

Dubossarsky, H., De Deyne, S., \& Hills, T. T. (2017). Quantifying the structure of free association networks across the life span. Developmental psychology, 53(8), 1560-1570.

Griffiths, T. L., Steyvers, M., \& Firl, A. (2007). Google and the mind: Predicting fluency with pagerank. Psychological science, 18(12), 1069-1076.

Hamel, R., \& Schmittmann, V. D. (2006). The 20-minute version as a predictor of the raven advanced progressive matrices test. Educational and Psychological measurement, 66(6), 1039-1046.

Healey, M. K., \& Kahana, M. J. (2016). A four-component model of age-related memory change. Psychological Review, 123(1), 23-69.

Kalbe, E., Kessler, J., Calabrese, P., Smith, R., Passmore, A., Brand, M. a., \& Bullock, R. (2004). Demtect: A new, sensitive cognitive screening test to support the diagnosis of mild cognitive impairment and early dementia. International journal of geriatric psychiatry, 19(2), 136-143. 
Kenett, Y. N., Beckage, N. M., Siew, C. S., \& Wulff, D. U. (2020). Cognitive network science: A new frontier. Complexity, 6870278.

Lehrl, S., Triebig, G., \& Fischer, B. (1995). Multiple choice vocabulary test mwt as a valid and short test to estimate premorbid intelligence. Acta Neurologica Scandinavica, 91(5), 335-345.

Morais, A. S., Olsson, H., \& Schooler, L. J. (2013). Mapping the structure of semantic memory. Cognitive science, 37(1), 125-145.

Naveh-Benjamin, M., Hussain, Z., Guez, J., \& Bar-On, M. (2003). Adult age differences in episodic memory: Further support for an associative-deficit hypothesis. Journal of Experimental Psychology: Learning, Memory, and Cognition, 29(5), 826-837.

Nelson, D. L., Zhang, N., \& McKinney, V. M. (2001). The ties that bind what is known to the recognition of what is new. Journal of experimental psychology. Learning, memory, and cognition, 27(5), 1147-59.

Psychology Software Tools, Inc. (2016). E-prime 3.0. https: //support.pstnet.com/

Ramscar, M., Hendrix, P., Shaoul, C., Milin, P., \& Baayen, H. (2014). The myth of cognitive decline: Non-linear dynamics of lifelong learning. Topics in cognitive science, 6(1), 5-42.

Siew, C. S., Wulff, D. U., Beckage, N. M., \& Kenett, Y. N. (2019). Cognitive network science: A review of research on cognition through the lens of network representations, processes, and dynamics. Complexity, 2108423.

Wechsler, D. (2008). Wechsler adult intelligence scale-fourth edition.

Wulff, D. U., De Deyne, S., Aeschbach, S., \& Mata, R. (2021, February 15). Understanding the aging lexicon by linking individuals' experience, semantic networks, and cognitive performance. https :// doi . org / 10 . 31234/osf.io/z3ebt

Wulff, D. U., De Deyne, S., Jones, M. N., Mata, R., \& Aging Lexicon Consortium. (2019). New Perspectives on the Aging Lexicon. Trends in Cognitive Sciences, 23(8), 686-698.

Wulff, D. U., Hills, T., \& Mata, R. (2018, October 29). Structural differences in the semantic networks of younger and older adults. https://doi.org/10.31234/ osf.io/s73dp 